\title{
Safety and tolerability of one-year intramuscular testosterone regime to induce puberty in older men with $\mathrm{CHH}$
}

\author{
Agnieszka Pazderska1,2, Yaasir Mamoojee', Satish Artham¹, Margaret Miller', Stephen G Ball3,4, \\ Tim Cheetham ${ }^{5,6}$ and Richard Quinton ${ }^{1,5}$ \\ 'Department of Endocrinology, Newcastle-upon-Tyne Hospitals, Newcastle upon Tyne, UK \\ ${ }^{2}$ Department of Endocrinology, St James's Hospital, Dublin, Ireland \\ ${ }^{3}$ Department of Endocrinology, Central Manchester University Hospitals, Manchester, UK \\ ${ }^{4}$ Department of Endocrinology, University of Manchester, Manchester, UK \\ ${ }^{5}$ Endocrine Research Group, Institute of Genetic Medicine, University of Newcastle-upon-Tyne, Newcastle upon Tyne, UK \\ ${ }^{6}$ Department of Paediatric Endocrinology \& Diabetes, Newcastle-upon-Tyne Hospitals, Newcastle upon Tyne, UK \\ Correspondence should be addressed to R Quinton: richard.quinton@ncl.ac.uk
}

\begin{abstract}
We present herein our 20-year experience of pubertal induction in apubertal older (median age 56 years; range 38.4-69.5) men with congenital hypogonadotrophic hypogonadism $(n=7)$ using a simple fixed-dose and fixed-interval intramuscular testosterone that we originally pioneered in relation to achieving virilisation of natal female transgender men. This regime was effective and well tolerated, resulting in complete virilisation by around 1 year after treatment initiation. No physical or psychological adverse effects were encountered in this group of potentially vulnerable individuals. There were no abnormal excursions of laboratory parameters and extended follow-up beyond the first year of treatment revealed remarkable improvements in bone density. We highlight advantages to both patients and physicians of this regime in testosterone-naïve older men with congenital hypogonadism and discourage the over-rigid application to such patients of treatment algorithms derived from paediatric practice in relation to the evaluation and management in younger teenagers with delayed puberty of uncertain cause.
\end{abstract}

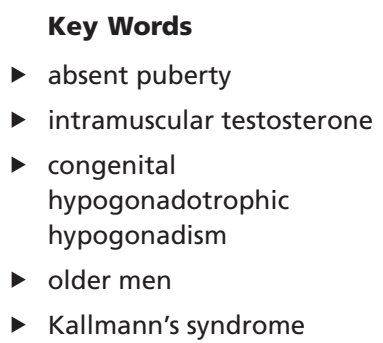

Endocrine Connections (2018) 7, 133-138

\section{Introduction}

Delayed puberty is defined by the absence of testicular development 2.5 s.D. later than the population mean typically 14 years of age in males (1). Although the majority of cases at age 14 have constitutional delay (CDP), the proportion of males with congenital hypogonadotrophic hypogonadism $(\mathrm{CHH})$ rises steeply thereafter, eventually comprising the universality of cases by the third decade of life (2). Whereas both primary and secondary $(\mathrm{CHH})$ ovarian insufficiencies can present with absent puberty in females, male hypogonadism in the context of absent puberty is almost invariably secondary $(\mathrm{CHH})(1)$.
Although sharing a common clinical presentation and biochemistry with $\mathrm{CDP}$, most $\mathrm{CHH}$ cases should be identifiable at baseline evaluation due to the presence of one or more red flags (e.g. congenital non-reproductive defects, particularly anosmia; history of testicular maldescent or neonatal micropenis, indicating likely absent minipuberty) (3), with the remainder being identifiable later during the process of pubertal induction through failure to normalise testicular volume with testosterone therapy (1).

Given the aforementioned factors, the diagnostic evaluation and management of pubertal delay should
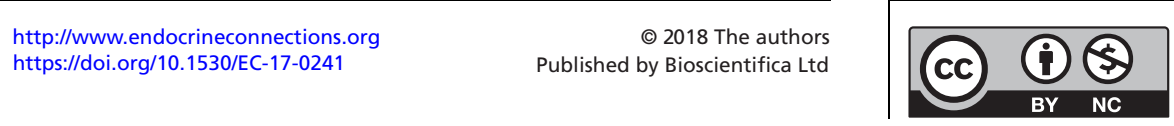

This work is licensed under a Creative Commons Attribution-NonCommercial 4.0 International License. 
be completed during the adolescent years, and yet this remains patently not the case. In line with the shared experiences of $\mathrm{CHH}$ men posted on online forums, two large $\mathrm{CHH}$ studies (one a traditional UK case notes-based series and another an international online patient survey), whose data collection was separated by over 20 years, found the median age of initiation of any meaningful treatment to be $18-19$ years $(4,5)$. The predominant reasons for treatment delay emerging from these studies are listed in Table 1, with almost all these coming into play for certain unfortunate individuals (6). In practice, the significance of $\mathrm{CHH}$ red flags is usually missed, both perinatally and in adolescence, leading to unnecessary delays in diagnosis and treatment (3).

$\mathrm{CHH}$ men with absent (around two-thirds of the cases) or arrested partial puberty (one-third) can thus present via a variety of routes in later life, e.g. during investigation of anaemia, muscle weakness, osteoporosis, infertility or sexual dysfunction, as a result of patients having finally summoned the courage to seek medical attention, or just serendipitously. Physicians facing an older man exhibiting congenital absence of puberty - possibly for the first time in their careers - notably lack published guidance on how best to initiate treatment (which product, how much and how quickly?). They understandably turn for guidance to their paediatrician colleagues, who encounter disorders of puberty far more commonly.

Paediatric practice and guidance are universally based upon the 'delayed puberty - query cause' (DPQC) pathway, which understandably emphasises the importance of reassurance and the avoidance of overmedicalisation, recommending low-dose, low-frequency pulses of testosterone if/when treatment is required (7). For adolescent boys, in whom delay is usually constitutional, testosterone treatment is typically initiated as low-dose intramuscular (IM) pulses, with the dose and/ or frequency tailored to allow clinical (testicular volume) and biochemical windows of opportunity for identifying endogenous gonadotrophin secretion. In teenagers with $\mathrm{CHH}$ or other organic hypogonadism, the aims are to recapitulate the normal tempo of male puberty over 2-3 years, whilst optimising linear growth potential and not masking evidence for endogenous gonadotrophindependent puberty should it emerge (1).

However, for those with 'totally obvious $\mathrm{CHH}^{\prime}$ (TOCHH - e.g. teenager with congenital anosmia or history of bilateral cryptorchidism, or any apubertal man in his third decade or beyond), feeding them into the DPQC pathway merely imposes unnecessary delays. Regimes envisaged and designed for teenagers with suspected CDP offer frustratingly slow progress for older $\mathrm{CHH}$ men, who have already attained near-final height, often exhibit segmental disproportion and, having experienced years of medical procrastination, express a reasonable desire to complete puberty without undue delay (6). Moreover, any opportunity for age-appropriate recapitulation of the normal tempo of puberty is long past.

Overvalued concerns that testosterone therapy might precipitate acute behaviour change - aggression, hypersexuality or priapism - in older apubertal men are surprisingly common among physicians, potentially further contributing to excessive caution with testosterone dosing in older men. Moreover, older men are more likely to harbour non-gonadal comorbidities for which there also seem to be lingering physician misconceptions in relation to the 'risks' of testosterone initiation. In addition, older men lacking capacity may no longer have a living relative for discussions of best interests. That $\mathrm{CHH}$ which is itself frequently associated with profound psychosocial issues adds another layer of complexity (8). Nevertheless, expert guidance is unequivocal, stating that age or disability per se should not constitute barriers to initiation of testosterone therapy in $\mathrm{CHH}$ (9).

The rarity of $\mathrm{CHH}$ (around 1 in 4000 males) (10) limits the accrual of experience even in the most specialist centres such as our own. The questions remain: what should the TOCHH pathway look like for a testosterone-naïve older man? And are there useful, and potentially more appropriate, experiences to be drawn from medical specialties other than paediatrics? Herein, we present our experience of a

Table 1 Factors underlying delayed initiation of age-appropriate treatment for $\mathrm{CHH}$ males.

Patient/parent factors

Physician factors/errors

\section{Delayed presentation}

Failure to attend follow-up visits

Failure to adhere to prescribed treatment

Belief that 'simple reassurance' is the only required first-line management for case of absent puberty beyond age 14

Belief that partial puberty (testicular volume $\geq 4 \mathrm{~mL}$ ) means that progression of puberty to completion is inevitable, so that patients can thus be safely discharged from follow-up Failure to identify or recognise the significance of 'red flag' features pointing to CHH over CDP Failure to prescribe clinically-meaningful doses of testosterone to adult men with confirmed $\mathrm{CHH}$ $\begin{array}{lr}\text { http://www.endocrineconnections.org } & \text { ○ } 2018 \text { The authors } \\ \text { https://doi.org/10.1530/EC-17-0241 } & \text { Published by Bioscientifica Ltd }\end{array}$
This work is licensed under a Creative Commons Attribution-NonCommercial 4.0 International License. 
simple 1-year (fixed-dose; fixed-interval) completion-ofpuberty regime with IM testosterone in older $\mathrm{CHH}$ men, presenting with untreated (or minimally treated) puberty. This regime has been significantly informed by our parallel clinical experience in achieving virilisation of testosteronenaïve (natal female) transgender males.

\section{Patients and methods}

Case records were reviewed for all $\mathrm{CHH}$ men $(n=7)$ who have been undergoing pubertal induction with testosterone at our centre since 1998 - aged 35 years or older. The diagnoses comprised Kallmann's syndrome $(n=5)$, normosmic $\mathrm{CHH}(n=1)$ and CHARGE syndrome $(n=1)$. Their clinical 'vignettes' are detailed in supplementary data (see section on supplementary data given at the end of this article), and offer some insights into the reasons for their extreme delay in presentation, but it was notable that one had major physical and learning impairments and another presented with life-changing injuries resulting from major self-harm episode influenced by low self-esteem.

Median age at treatment initiation was 56 years (range 38.4-69.5). Treatment over the first year was with IM Sustanon $250 \mathrm{mg}$ administered monthly $(n=1)$, or testosterone undecanoate $1 \mathrm{~g}$ (Nebido/Reandron-TU) administered around four months apart $(n=6)$ - omitting the usual loading dose recommended at 6 weeks. All patients additionally received daily supplementation with calcium and cholecalciferol.

The data presented in this manuscript originated not from a registered clinical trial, but from an internal audit of our established clinical practice. Therefore, formal institutional ethical approval was not required, because non-identifiable data are used with all subjects or guardians consenting to the inclusion of the data being presented.

Statistical analysis was performed using SPSS for Windows version 22 (IBM SPSS Statistics 22). Data presented are expressed as medians with interquartile ranges. Pre- and post-treatment parameters were compared using the Mann-Whitney $U$-test.

\section{Results}

Longitudinal data detailing pubertal staging, laboratory parameters and bone mineral density are shown in Table 2. All patients completed puberty within a year of treatment initiation. Of those who were able to express an opinion $(n=6)$ were very satisfied with the pace of change and final outcome, with similar views being expressed by the carers of patient 5 . As expected for a group of men with $\mathrm{CHH}$, rather than DPQC, none developed any features of endogenous puberty, such as an increase in trough LH concentration greater than $2.0 \mathrm{IU} / \mathrm{L}$, or testicular enlargement greater than $4 \mathrm{~mL}$.

During testosterone treatment, there were no adverse effects recorded, except for rapidly progressive male-pattern baldness $(n=1)$. Despite the presence of major cognitive or psychological issues in three out of seven men prior to initiation of testosterone therapy, there were neither adverse psychological events recorded nor any worsening in mood or behaviour noted during treatment. In the CHARGE patient-lacking capacity, DISDAT (Disability Distress Assessment Tool (http://prc.coh.org/PainNOA/ Dis\%20DAT_Tool.pdf) scores regularly calculated by experienced disability care staff remained stable.

Trough serum testosterone concentrations during treatment did not show any wide excursions and, as expected, therapy resulted in significantly increased haemoglobin concentration $(122 \pm 29-157 \pm 8 \mathrm{~g} / \mathrm{L})$ and haematocrit $(37 \% \pm 9-45 \% \pm 5)-(P<0.05)$, but without any supraphysiological rise. Patient numbers were necessarily insufficient in respect of detecting significant changes in lipid profile, PSA or HbA1c, but the raw data appeared very reassuring. Follow-up beyond the initial year of treatment revealed a median increase of $49 \%$ in lumbar spine bone mineral density: median $t$-score rising from minus $3.5 \pm 1.0$ at baseline to minus $1.8 \pm 1.2$ after a median 6 years of testosterone therapy $(P<0.05)$.

\section{Discussion}

Aside from isolated reports and conference abstracts, this is the first systematic evaluation of pubertal induction (methods and outcomes) in testosterone-naïve older men with congenital hypogonadism. Subject to necessarily limited patient numbers accruing to a less common presentation of a rare disease, we have demonstrated that completion of puberty over 1 year with IM testosterone can be achieved with patients' satisfaction and without significant adverse events in older apubertal men with $\mathrm{CHH}$; thereafter, there are sustained major improvements in bone mineral density, even as far as the sixth and seventh decades of life. Pubertal induction in this context can be achieved using either short- or long-acting IM testosterone injections, but depot-TU conveniently minimises outpatient visits.

Although baseline osteopaenia or osteoporosis is entirely expected in these patients, as a result of congenital http://www.endocrineconnections.org https://doi.org/10.1530/EC-17-0241
(C) 2018 The authors Published by Bioscientifica Ltd
This work is licensed under a Creative Commons Attribution-NonCommercial 4.0 International License. 
H.

11.

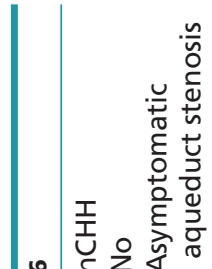

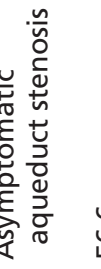

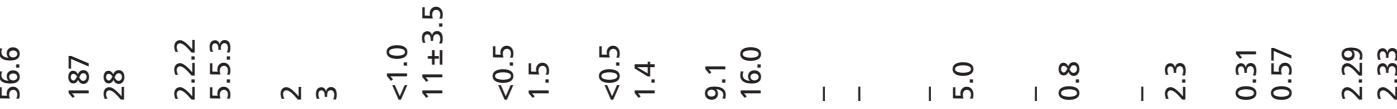

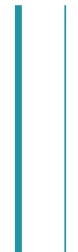

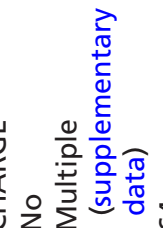

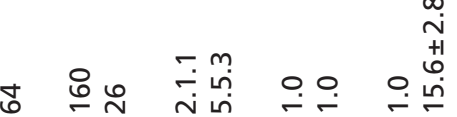

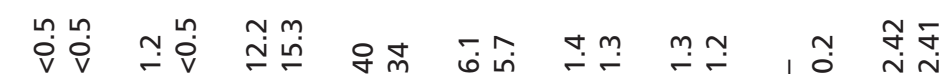

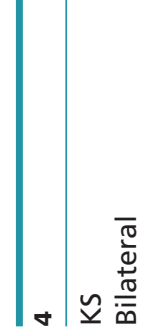
离

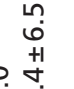

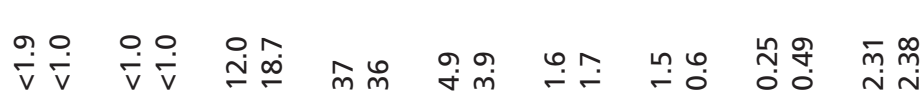

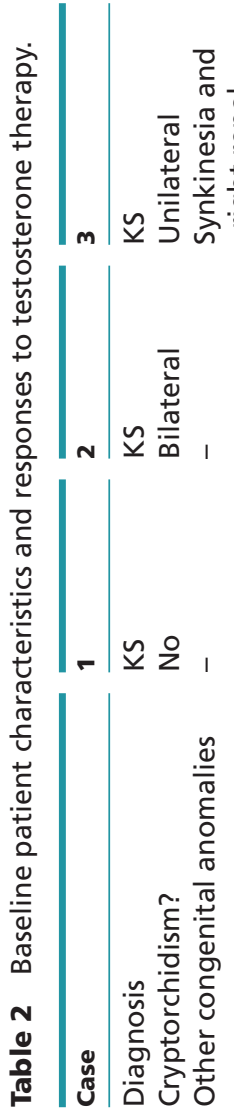




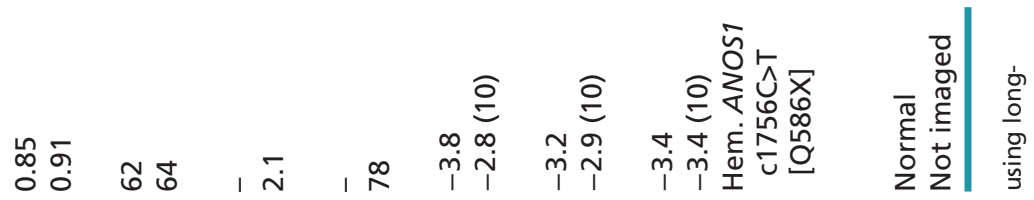

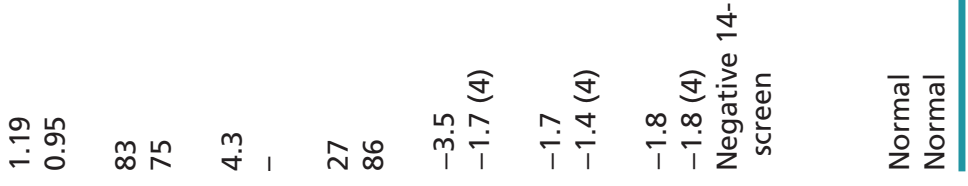

농

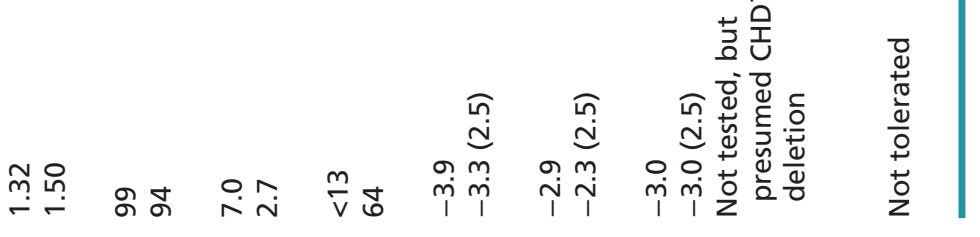

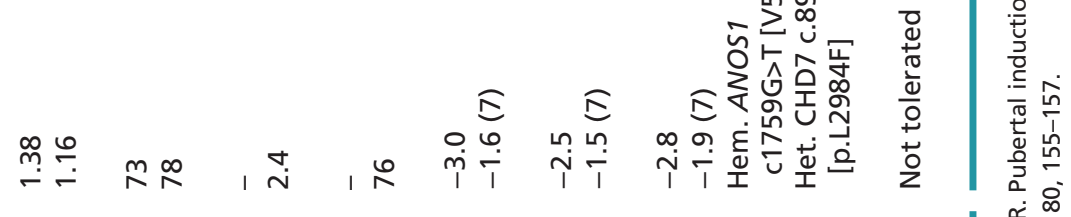

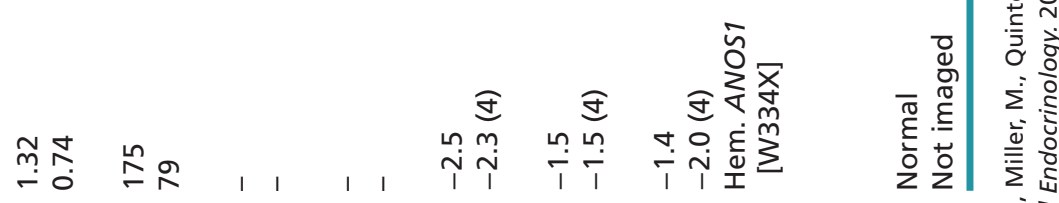

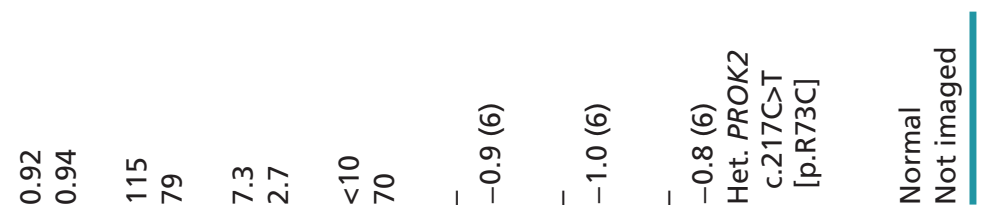

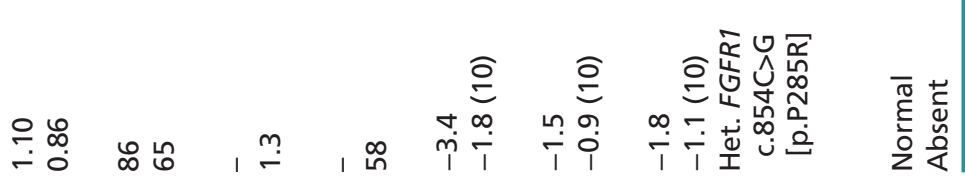

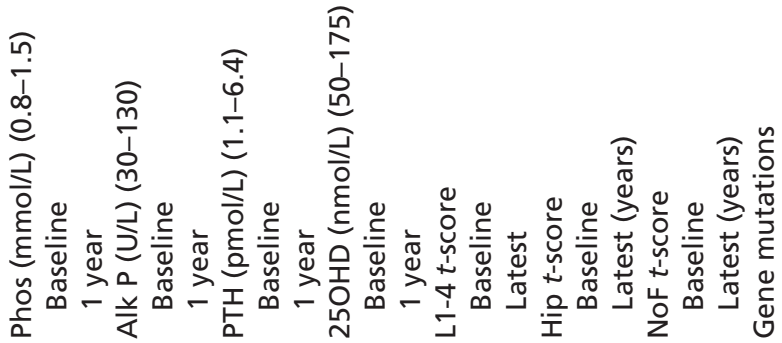

http://www.endocrineconnections.org https://doi.org/10.1530/EC-17-0241 (c) 2018 The authors Published by Bioscientifica Ltd
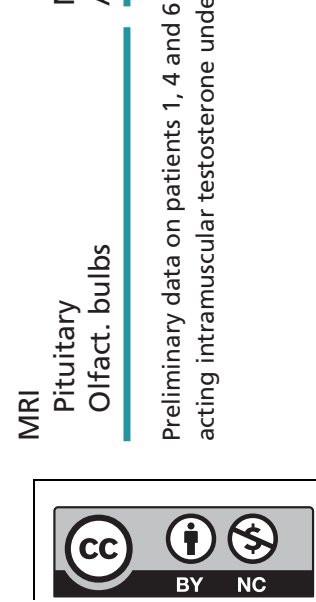

This work is licensed under a Creative Commons Attribution-NonCommercial 4.0 International License. 
androgen deficiency, our protocol is to defer adjuvant antiresorptive therapy until anabolic (testosteronedriven) improvements in bone mineral density have plateaued. We would, of course, revisit this strategy in the event of intercurrent spinal fracture for which parenteral antiresorptives may also be beneficial for pain control and bone remodelling, but have not yet faced this scenario in this group of patients.

Despite widespread physician concerns about potential behaviour disturbance in testosterone-naïve adult men undergoing physiological normalisation of serum testosterone, literature review failed to uncover any evidence to underpin these concerns. This reflects our experience reported herein, which is in line with that of clinicians treating much larger numbers of older testosterone-naïve transgender males with cross-hormone regimes. Transgender men have a particularly high baseline prevalence of psychological/psychiatric disease and are likewise virilised fairly rapidly, and yet significant testosterone-related behaviour disturbance has not been observed in this population $(11,12)$.

However, we emphasise that the regime described herein delivers just $2 \mathrm{~g}$ of TU over the first 32 weeks of therapy and, in testosterone-naïve men, would strongly caution against using standard TU initiation regimes as per the product datasheet comprising two injections spaced 6 weeks apart followed by 12 -weekly maintenance injections (i.e. cumulative $4 \mathrm{~g}$ of TE over the first 30 weeks of therapy).

\section{Conclusions}

We suggest that clinicians avoid reflexly applying DPQC management algorithms to older testosterone-naïve men (or younger ones with 'red flags' for $\mathrm{CHH}$ ). We instead commend to them this simple and well-tolerated pubertal induction regime using fixed-dose and fixed-interval IM testosterone. In respect of potential psychological issues experienced by older testosterone-naïve men during pubertal induction, we believe that the more transferrable lessons emerge from the transgender - rather than the adolescent - literature.

\section{Supplementary data}

This is linked to the online version of the paper at https://doi.org/10.1530/ EC-17-0241.

\section{Declaration of interest}

The authors declare that there is no conflict of interest that could be perceived as prejudicing the impartiality of the research reported.

\section{Funding}

This research did not receive any specific grant from any funding agency in the public, commercial or not-for-profit sector.

\section{References}

1 Palmert MR \& Dunkel L. Clinical practice. Delayed puberty. New England Journal of Medicine 2012366 443-453. (https://doi org/10.1056/NEJMcp1109290)

2 Dunkel L \& Quinton R. Transition in endocrinology: induction of puberty. European Journal of Endocrinology 2014170 R229-R239. (https://doi.org/10.1530/EJE-13-0894)

3 Quinton R, Mamoojee Y, Jayasena CN, Young J, Howard S, Dunkel L, Cheetham T, Smith N \& Dwyer AA. Society for Endocrinology UK guidance on the evaluation of suspected disorders of sexual development: emphasizing the opportunity to predict adolescent pubertal failure through a neonatal diagnosis of absent minipuberty. Clinical Endocrinology 201786 305-306. (https://doi.org/10.1111/ cen.13257)

4 Quinton R, Duke VM, Robertson A, Kirk JM, Matfin G, de Zoysa PA, Azcona C, MacColl GS, Jacobs HS, Conway GS, et al. Idiopathic gonadotrophin deficiency: genetic questions addressed through phenotypic characterization. Clinical Endocrinology 200155 163-174. (https://doi.org/10.1046/j.1365-2265.2001.01277.x)

5 Dwyer AA, Tiemensma J, Quinton R, Pitteloud N \& Morin D. Adherence to treatment in men with hypogonadotrophic hypogonadism. Clinical Endocrinology 201786 377-383. (https://doi. org/10.1111/cen.13236)

6 Smith N \& Quinton R. Kallmann syndrome. BMJ 2012345 e6971. (https://doi.org/10.1136/bmj.e6971)

7 Howard S \& Dunkel L. Sex steroid and gonadotropin treatment in male delayed puberty. Endocrine Development 201629 185-197. (https://doi.org/10.1159/000438891)

8 Dwyer AA, Quinton R, Pitteloud N \& Morin D. Psychosexual development in men with congenital hypogonadotropic hypogonadism on long-term treatment: a mixed methods study. Sexual Medicine 20153 32-41. (https://doi.org/10.1002/sm2.50)

9 Boehm U, Bouloux PM, Dattani MT, de Roux N, Dodé C, Dunkel L, Dwyer AA, Giacobini P, Hardelin JP, Juul A, et al. Expert consensus document: European Consensus Statement on congenital hypogonadotropic hypogonadism - pathogenesis, diagnosis and treatment. Nature Reviews Endocrinology 201511 547-564. (https:// doi.org/10.1038/nrendo.2015.112)

10 Fromantin M, Gineste J, Didier A \& Rouvier J. Impuberism and hypogonadism at induction into military service. Statistical study. Problemes Actuels D'Endocrinologie et de Nutrition 197316 179-199.

11 Gooren LJ. Management of female-to-male transgender persons: medical and surgical management, life expectancy. Current Opinion in Endocrinology, Diabetes and Obesity 201421 233-238. (https://doi. org/10.1097/MED.0000000000000064)

12 Gooren LJ, Sungkaew T, Giltay EJ \& Guadamuz TE. Cross-sex hormone use, functional health and mental well-being among transgender men (Toms) and Transgender Women (Kathoeys) in Thailand. Culture, Health and Sexuality 201517 92-103. (https://doi. org/10.1080/13691058.2014.950982)

Received in final form 16 November 2017

Accepted 27 November 2017
This work is licensed under a Creative Commons Attribution-NonCommercial 4.0 International License. 